\title{
Functional Topography in Cat Area 18
}

\author{
M. S. Cynader, ${ }^{1,2}$ N. V. Swindale, ${ }^{1}$ and J. A. Matsubara ${ }^{3}$ \\ Departments of ${ }^{1 P}$ sychology, ${ }^{2}$ Physiology and Biopinysics, and ${ }^{3}$ Anatomy, Dalhousie University, Halifax, B3H 4J1, Canada
}

\begin{abstract}
Using closely spaced microelectrode penetrations, we have mapped the representation of the visual field and of several functional response properties on the surface of cat area 18. The representation of the visual field was anisotropic, with the magnification factor for vertical visual space being about 2-5 times greater than that for horizontal. The topographic anisotropy was paralleled by an anisotropy in the cortical point-spread function, a measure of the distribution in the cortex of cells with overlapping receptive-field centers. The spread of this distribution (measured as twice the standard deviation) was positively correlated with the magnification factor anisotropy, averaging about $0.6 \mathrm{~mm}$ along the mediolateral (M-L) axis and $1.2 \mathrm{~mm}$ along the anteroposterior (A-P) axis.
\end{abstract}

Units with similar response properties were clustered across the cortical surface, but different response features were laid out in different ways. For units with orientations within a $90^{\circ}$ range, the clusters took the form of branching bands, with a center to center spacing of $1.25 \pm 0.13 \mathrm{~mm}$. These bands ran from postero-medial to anterolateral across the cortical surface, roughly perpendicular to the $17 / 18$ border. Units with similar preferred eye input were laid out in patches without a well-defined direction of elongation, and with a less well-defined periodicity that averaged $1.86 \pm$ $0.75 \mathrm{~mm}$. Local correlations in direction preference extending over distances of $300 \mu \mathrm{m}$ were found, together with frequent $180^{\circ}$ differences in the direction preferences of units close together. The organization of the map of orientation preference, and the way in which direction selectivity is overlaid on this map, is discussed in more detail in the accompanying paper (Swindale et al., 1987).

No obvious structural relationships between the ocular dominance patches and the pattern of iso-orientation domains were observed. There were, however, obvious interrelationships between the topographic map and other functional response properties. The iso-orientation bands ran approximately perpendicular to the direction in which both the point-spread function and magnification factor were elongated. This will have the effect of maximizing the allocation of a full range of orientations by the cortex to each location in visual space.

In the last 2 decades, the nature of the 3-dimensional organization of the cortex has become increasingly clear. In the visual cortex in particular, the discovery of columnar systems for ori-

\footnotetext{
Received June 10, 1986; revised Sept. 15, 1986; accepted Oct. 2, 1986.

We are especially grateful to Julia Hickey and Lori Colpitts for their help at all stages of these experiments. We also thank Gordon Troop. Nick McGraw, and Ferenc Stefani for technical and photographic assistance. Rhone-Poulenc Pharma generously donated a supply of gallamine triethiodide. The research was supported by Grants PG-29 from the MRC of Canada and A9939 from the NSERC of Canada to M.S.C.

Correspondence should be addressed to M. S. Cynader, Dept. of Psychology, Dalhousie University, Halifax, B3H 4J1, Nova Scotia, Canada.

Copyright (C) 1987 Society for Neuroscience $0270-6474 / 87 / 051401-13 \$ 02.00 / 0$
}

entation and ocular dominance, superimposed on a retinotopic map, has led to questions about the way in which these various maps are organized on the cortical surface, and about how they are arranged in relation to one another. The tangential organization of functional properties in the cortex has been examined using a variety of anatomical and physiological methods, including 2-deoxyglucose autoradiography (Kennedy et al., 1976; Hubel et al., 1978; Albus, 1979; Humphrey et al., 1980; Singer, 1981), transneuronal transport of tritiated amino acids (Wiesel et al., 1974; Hubel et al.,1977; Shatz et al.,1977), tangentially directed electrode penetrations (Hubel and Wiesel, 1974a, b; Albus, 1975a, b), and by making a series of closely spaced electrode penetrations perpendicular to the cortical surface (Hubel and Wiesel, 1963). Each of these approaches has its advantages and disadvantages. The 2-deoxyglucose method enables one to visualize the layout of units with particular functional properties, but it has proved difficult to relate the various functional properties to the retinotopic map and to one another. Long tangential penetrations through the cortex make it possible in principle to derive all the relevant properties and their interrelationships with high resolution, but the cortical area that can be studied is small, and it is difficult to generalize from 1-dimensional penetrations to the 2-dimensional layout of response properties that is of interest.

We have adopted the approach of making a large number of closely spaced electrode penetrations into the superficial layers of the cortex. At each penetration site we determined response properties, including orientation, direction, and ocular preference. We also measured receptive-field size and location. We chose the part of cat area 18 containing the lower visual-field representation for these investigations since it provides a naturally flattened surface, which is convenient for mapping (Fig. 1). We studied (1) the local organization of the retinotopic map on the cortex, measuring parameters such as magnification factor, its anisotropy, and the amount of receptive-field scatter; (2) how the response properties of orientation preference, direction selectivity, and ocular dominance were mapped on the surface of the cortex; and finally (3) the relationship of these response properties to one another and to the retinotopic map.

In accompanying papers we analyze the layout of orientation and direction preferences in area 18 in greater detail (Swindale et al., 1987), and the distribution and physiological correlates of intrinsic cortical connections, as revealed by localized injections of tracers into physiologically identified regions of our maps (Matsubara et al.,1987).

\section{Materials and Methods}

Thirty-eight adult cats were used in these experiments. Our methods of obtaining single-unit recordings from the visual cortex of acutely prepared, anesthetized cats were the same as those used previously (Cynader and Mitchell, 1980; Cynader et al., 1984). Cats were initially anesthetized with intravenous pentobarbital sodium, a tracheotomy was performed, and the animals paralyzed with intravenous gallamine triethiodide. Atropine $(0.2 \mathrm{mg}$, i.v. $)$ and dexamethasone $(0.3 \mathrm{mg}$, s.c.) were 
Figure 1. A surface view of area 18, photographed at the beginning of an experiment. The surface pattern of blood vessels was used as a reference for establishing the position of each electrode penetration. Scale bar, $1 \mathrm{~mm}$. Anterior $(A)$ is down and medial $(M)$ is to the right. All subsequent diagrams of the cortical surface are from this perspective.

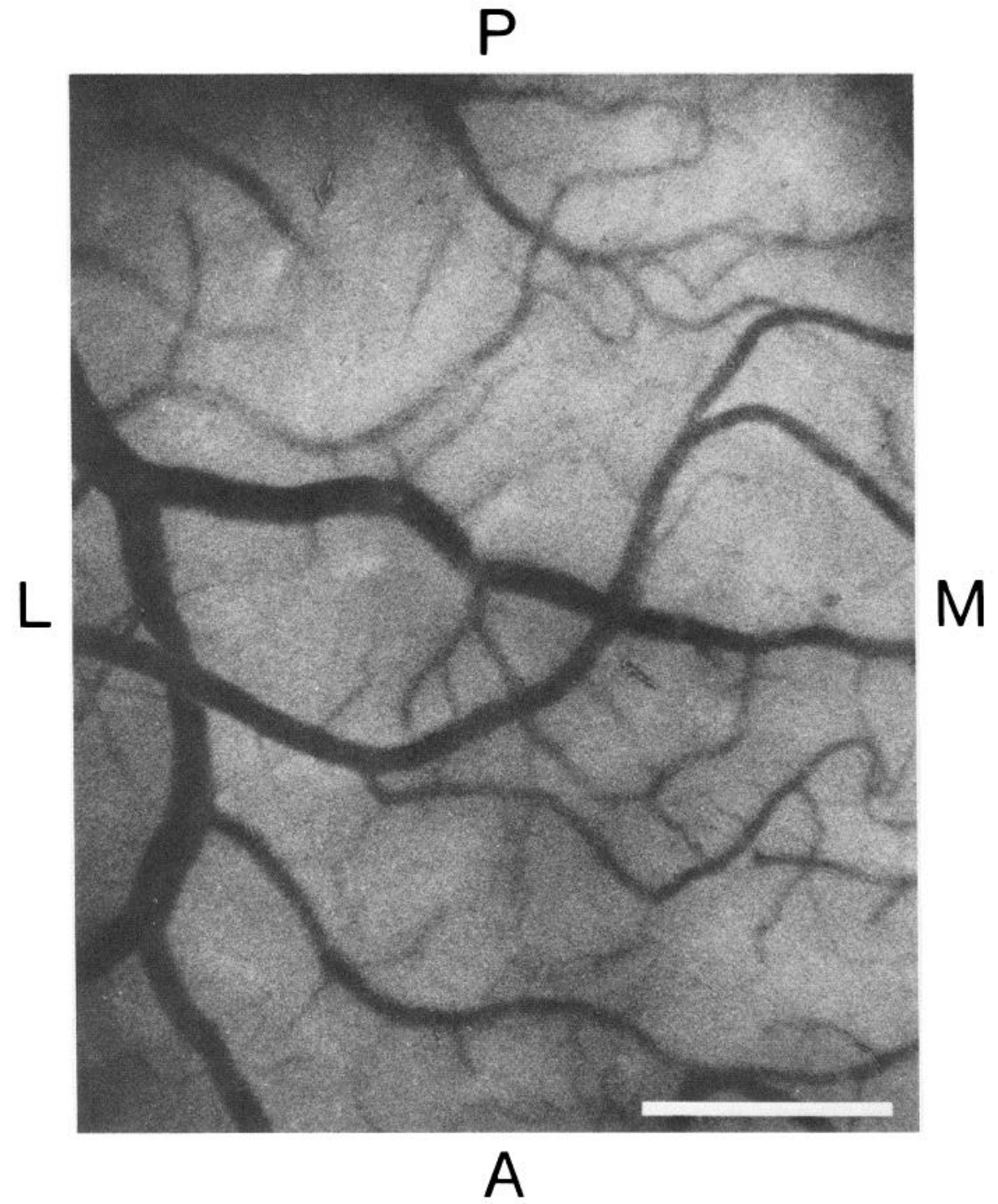

administered to decrease salivation and prevent brain oedema, respectively.

During recording, light anesthesia was maintained by artificially ventilating the animal with a mixture of $\mathrm{N}_{2} \mathrm{O}$ and $\mathrm{O}_{2}$ (70:30), and intravenous anesthesia was discontinued. Pressure points and wounds were infiltrated with a long-lasting local anesthetic (Marcaine, 0.25\%). The animal's body temperature was maintained near $38^{\circ} \mathrm{C}$ with a thermostatically controlled heating pad, and end-tidal $\mathrm{pCO}_{2}$ was monitored continuously and maintained near $4.0 \%$ by varying the rate of an artificial respiration pump. EEG and EKG were monitored routinely. Contact lenses were chosen by retinoscopy to focus the eyes on a tangent screen $145 \mathrm{~cm}$ distant; the lenses contained $4 \mathrm{~mm}$ artificial pupils to decrease scattered light and increase depth of focus.

A craniotomy approximately $8 \times 12 \mathrm{~mm}$ in size was made over the lower-field representation of area 18 . The dura was resected and a Davies chamber sealed onto the skull with dental acrylic cement and skull screws. By providing a hydraulically sealed volume around the exposed brain surface, which remained visible through a Plexiglas plate, the Davies chamber substantially eliminated pulsations of the cortex caused by respiratory movements and heartbeats. The brain surface and a 1 $\mathrm{mm}$ scale bar placed adjacent to it were photographed using a $35 \mathrm{~mm}$ camera with a "macro" lens and Polaroid instant slide film. An enlarged image of this slide (Fig. 1) was projected onto acetate film and the sites of electrode penetrations, as defined by their positions relative to the pattern of blood vessels on the surface of the cortex, were marked as the experiment progressed. The recording electrode was manipulated while it and the cortical surface were viewed through a dissecting microscope, and was positioned so as to avoid damage to blood vessels on entry into the cortex. A significant advantage of using an initial photograph of the cortex and blood vessels as a reference for establishing the positions of recording sites is that subsequent distortions or movements of the cortex will not affect the accuracy with which cortical position is measured (as it would if micrometer readings on the microelectrode holder were used instead).

The electrodes were made of finely tapered platinum-iridium wire, coated with glass (Wolbarsht et al., 1960), and driven through the cortex with a microprocessor-controlled stepper motor. Their impedance was typically $0.3-0.6 \mathrm{M} \Omega$, and they had an exposed uninsulated tip length of 30-50 $\mu \mathrm{m}$. Shaft diameter was approximately $80 \mu \mathrm{m}$ at a distance of $500 \mu \mathrm{m}$ from the tip, and caused no damage that could be detected histologically. Penetrations were approximately normal to the surface and were made to a depth of $400-700 \mu \mathrm{m}$. All penetrations were parallel to one another. Neural activity of single units or small clusters of cells was amplified, band-pass filtered, and monitored on an oscilloscope and audiomonitor.

Since measurement of receptive-field scatter was an important aim in our experiments, particular care was taken to minimize the sources of error involved in making accurate determinations of receptive-field position and size. These included errors inherent in making hand plots of receptive-field size based on auditory judgments of response, and errors associated with residual small movements of the eyes that occur under paralysis (Chow, 1968; Bishop et al., 1971). It was noticed, for 


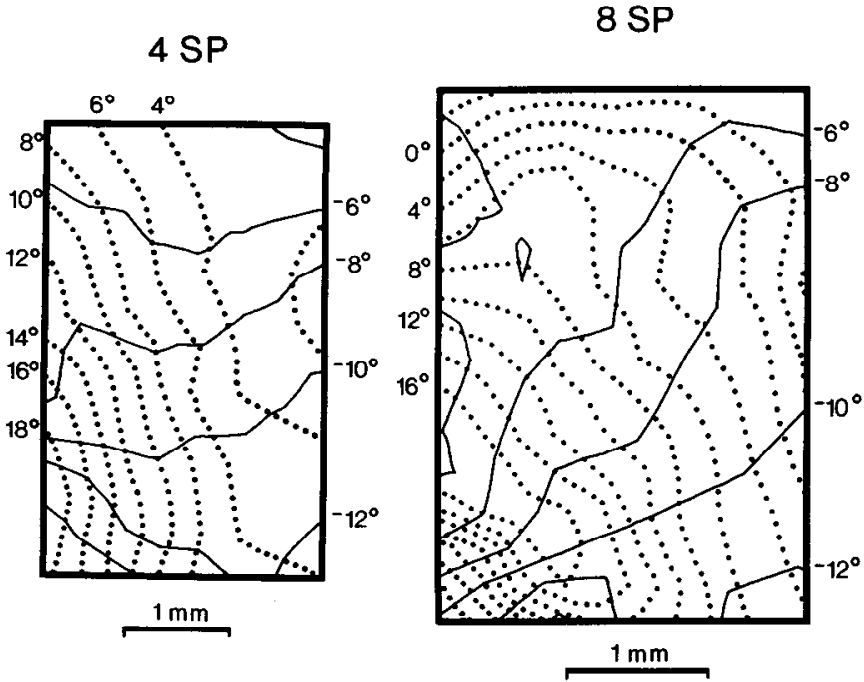

$12 \mathrm{SP}$
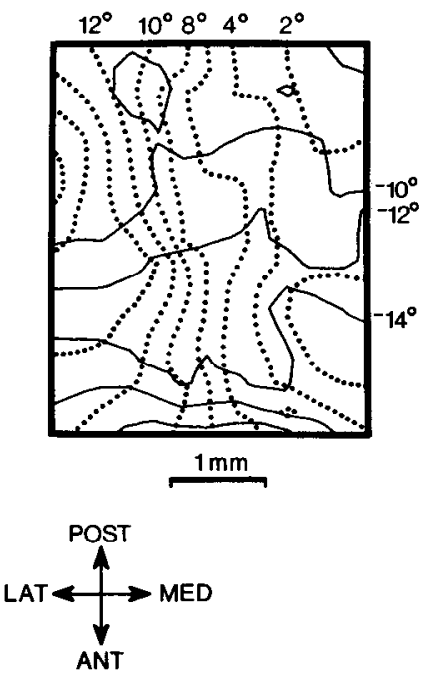

Figure 2. Contour plots of the retinotopic map on the surface of area 18 in 3 different animals. Continuous lines are iso-elevation contours and dotted lines are iso-azimuthal contours. The interval between both sets of contours is $2^{\circ}$ of visual angle. See text for further description. example, that overall responsivity affected the determination of receptive-field boundaries, although receptive-field-center location stayed relatively constant. For this reason we chose to concentrate our analysis on receptive-field-center position rather than on receptive-field size. Repeated plots of responses at the same cortical locus and plots by 2 observers yielded inaccuracies of only about $0.5^{\circ}$ in the determination of receptive-field position. Residual movements of the eyes were controlled for by making frequent repeated measurements, with a reversing ophthalmoscope, of the optic disks and the area centralis location. To more accurately detect residual eye movements, the position of an easily identifiable retinal landmark, such as the intersection of 2 blood vessels, was also plotted. Measurements of receptive-field location were subsequently corrected for such movements, which normally amounted to no more than $\pm 2^{\circ}-3^{\circ}$ during the course of an experiment.

Once visual responses were evoked at a given location, we mapped the receptive-field location and size onto the tangent screen. We also noted the preferred orientation, ocularity, and direction for the units in question. All responses were judged by listening to the audiomonitor and no quantitative analytic methods were used. We generally accumulated data from between 80 and 150 points in a 2 d experiment. Penetrations were made in a quasiregular grid pattern with an average spacing of about $300 \mu \mathrm{m}$. The area mapped was normally about $3 \times 4$ $\mathrm{mm}$ in extent.

Multiunit responses recorded in the cortex contain, in principle, contributions from thalamocortical axons as well as cortical cells. However, at the intracortical depths (almost always layers II and III) from which the data were derived, a direct contribution of thalamocortical input would be weak or absent. With few exceptions, all recorded neurons at given sites had common response properties. When single units were recorded in the sample, or, as was often the case, when units were evident in the multiunit cluster, the response of the isolated unit paralleled the response of unresolved units in the background. An advantage of the multiunit recording method is that exceptionally responding units or clusters of units are readily detected, i.e., it is very evident when all recorded responses at a given recording site do or do not have identical response properties.

A number of the maps were found to lie close to, or to straddle the border between, areas 17 and 18 , as judged by the presence of many receptive fields centered on the vertical meridian at one edge of the map. These maps were excluded from the analysis of magnification factor and receptive-field scatter (these data will be presented in a subsequent publication), but not from the analysis of the other properties reported in this and the 2 accompanying papers.

To mark selected recording sites for anatomical correlations, we injected wheat germ agglutinin-conjugated horseradish peroxidase or concanavalin A for use as tracers, and injected fluorescent dyes or rhodamine-labeled latex microspheres for use as landmarks (Matsubara et al., 1987).
Data analysis. For each recording location in any particular map, the following data were stored on disc in a computer: the penetration number, the cortical coordinates of the recording site in millimeters, the receptive-field position in degrees relative to the area centralis, receptive-ficld size, preferred oricntation, preferred direction (or a value signifying no direction preference), and ocular dominance (measured on Hubel and Wiesel's (1962) 7-point scale). These data were then subjected to various kinds of analysis, including (1) determination of the directions of iso-elevation, iso-azimuthal, and iso-eccentricity contours in the visual field, as mapped onto the cortical surface; (2) examination of the associated magnification factors of these contours; (3) measurement of receptive-field scatter, i.e., the extent to which the retinotopic map on the cortex deviated from perfect smoothness, a measurement that we refer to as the cortical point-spread (or scatter) function; (4) Fourier spectral analysis of the orientation, ocular dominance, and direction maps, performed to determine the regularity and periodicity in the maps of these properties. Further details of the quantitative methods used in the receptive-field-position analysis are given in Results, and a detailed analysis of the orientation and dircction maps is presented in an accompanying paper (Swindale et al., 1987).

\section{Results}

We group our results into 3 main sections, dealing first with the retinotopic map and its disorder, then with the tangential arrangement of preferred orientation, direction, and ocularity, and finally with the relationships between the functional response properties and the retinotopic map.

\section{Retinotopic map}

The representation of the visual field over the area of cortex studied is illustrated for 3 animals in Figure 2. Each panel represents the cortical surface, the top of the pancl bcing posterior and the right-hand side medial. The dotted lines running roughly anterior-posterior across the cortical surface represent the isoazimuthal contours of the visual field. The solid lines running approximately medial to lateral represent the iso-elevation contours. To obtain these contours, the data were first smoothed by convolution with a Gaussian kernel to obscure local variability. The kernel had a space constant of about $300 \mu \mathrm{m}$. The interval between contour lines represents $2^{\circ}$ of visual angle. The region of visual space from which all of the maps come extends $5^{\circ}-15^{\circ}$ below the horizontal meridian, and from $0^{\circ}$ to $20^{\circ}$ ec- 


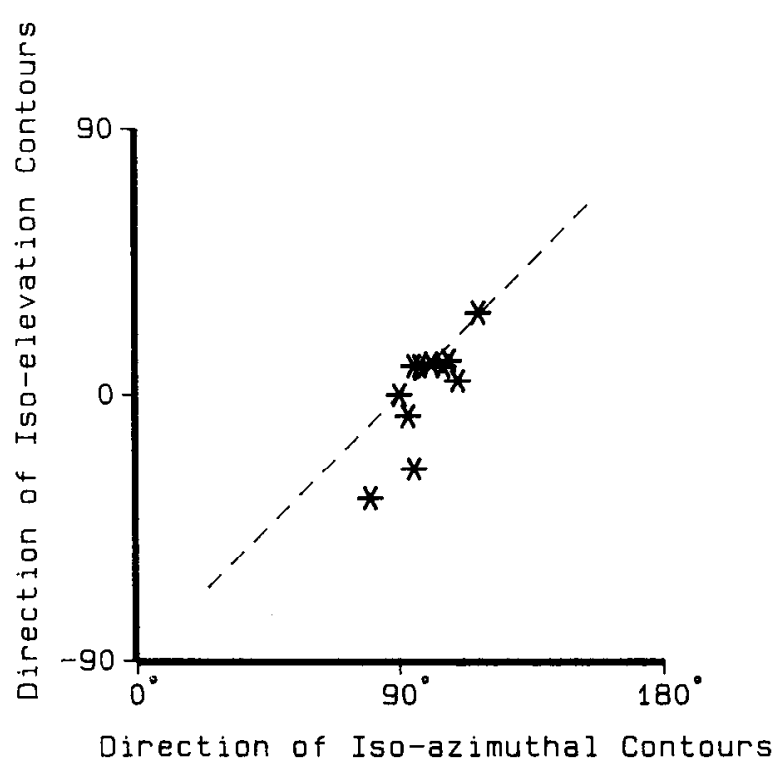

Figure 3. Scatter plot graphing the directions across the cortex in which the iso-elevation and iso-azimuthal contours run. Directions are measured relative to the mediolateral axis on the right-hand side of the brain, as though each map came from this hemisphere. Each point is a measurement from 1 animal. If the contours intersected exactly at right angles, each point would lie somewhere on the dashed line.

centric to the vertical meridian. Figure 2 shows that, in all 3 animals, there is a marked anisotropy in the cortical magnification factors for horizontal and vertical components of visual space. Thus a given vertical distance in visual space maps onto a greater distance in the cortex than does an equivalent horizontal distance in visual space. This anisotropy varies with eccentricity and from animal to animal (see Fig. $7 b$ ), but is a consistent feature of all of our maps.

A more detailed analysis than that illustrated by Figure 2 was performed to measure the horizontal and vertical axis magnification factors, and the angle at which the lines of iso-elevation and iso-azimuth run across the cortex. This analysis is illustrated in Figure 6 and is based on the premise that, for a small map, the lines of iso-elevation and iso-azimuth will be approximately straight and evenly spaced across the cortex. Inspection of Figure 2 shows that this assumption is a good one, except as concerns a few points at the extreme edges of some of the maps. Scatter graphs were constructed (of which those in Fig. 6 are examples), in which receptive-field elevation or azimuth was plotted against a single component of cortical distance for a particular direction of scan across the cortical surface. Regression analysis was then uscd to fit a straight line to the data and compute the goodness of the fit. This was performed for a variety of scan angles, and the angle giving the best-fitting regression line, measured to the nearest $5^{\circ}$, was considered the best estimate of the direction in which the contour lines for elevation or azimuth run. The slope of the line, in millimeters per degree, gave the magnification factor. Correlation coefficients for the regression were typically in the range $0.8-0.95$ for the best scan angles, and decreased to values of less than 0.1 for angles of scan orthogonal to this.

This analysis was performed for 11 maps and the results are summarized in Figures 3 and 4. Figure 3 plots the directions of the iso-elevation and iso-azimuthal contours in relation to the cortical A-P axis. These directions (and other directions relative

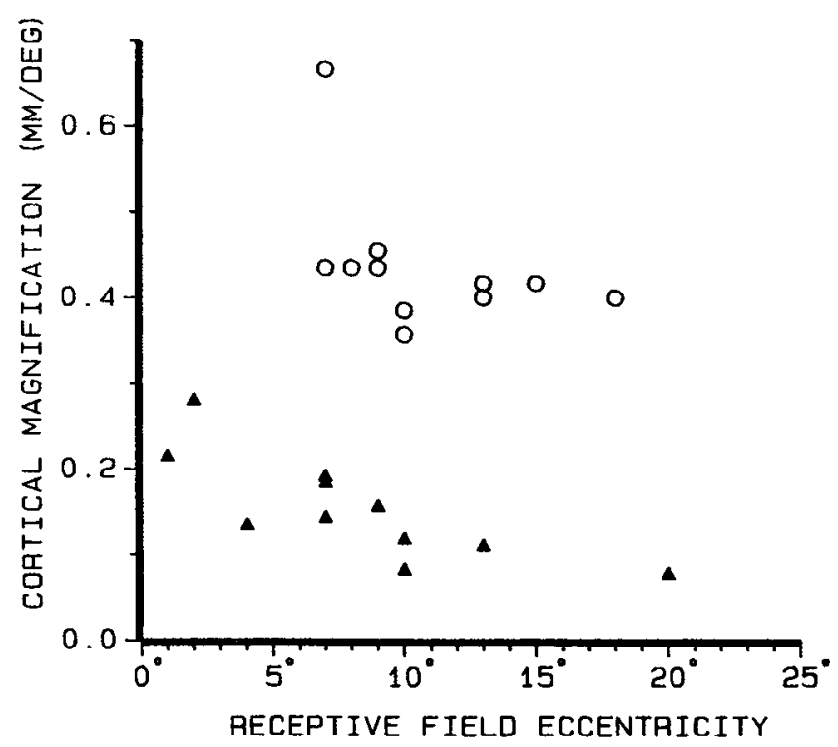

Figure 4. Open circles represent the vertical magnification factor (expressed as millimeters of cortex/degree of elevation) graphed against the mean vertical eccentricity (elevation) of the map. Filled triangles represent the horizontal magnification factor graphed against the mean horizontal cccentricity (azimuth) of the map. Each point is data from 1 animal.

to the cortical surface referred to in this paper) are expressed relative to the mediolateral axis, anticlockwise-positive, and as though the maps all came from the right hemisphere of the brain. Note that these directions are clustered around $0^{\circ}$ and $90^{\circ}$, respectively, and that there is a tendency for the intersection angle to remain at $90^{\circ}$ despite differences in the overall orientation of the retinotopic map relative to the surface of the cortex.

Figure 4 plots the cortical magnification factor (open circles) for the vertical component of visual space (in millimeters per degree) against the mean vertical component of eccentricity of the map for 11 animals. The corresponding magnification factor for horizontal visual space (filled triangles) against the horizontal component of eccentricity is also plotted. The average magnification factor for the horizontal component of visual space decreases with eccentricity. This reflects the well-known tendency of cortical maps to emphasize the representation of the visual-field midline in relation to the contralateral periphery. The relationship is approximately reciprocal, with a slope of 0.5 $\mathrm{mm}^{-1}$. For the data relating vertical magnification factor to receptive-field eccentricity, the existence of a similar relationship is equivocal. The position of one data point (uppermost on the graph) suggests, however, that magnification factor may increase at eccentricities of $5^{\circ}$ or less. The amount of anisotropy, expressed as the ratio between the magnification factors for horizontal and vertical, varied between 1.9 and 5.4 in 11 different maps (Fig. 7b).

Despite the magnification anisotropy in the representation of horizontal and vertical visual axes, the iso-elevation and isoazimuthal contours of the visual field run approximately perpendicular to each other when mapped on the cortical surface just as, by definition, they do in the real world. It is interesting that anisotropic mappings (which are nonconformal; see Schwartz, 1980) will not in general preserve angular relationships between axes in the retinotopic and cortical frames of reference. For example, Figure $5 a$ shows another view of the retinotopic map of 1 of the 3 animals in Figure 2, but now the 
contour lines represent visual-field lines $45^{\circ}$ to the right and to the left of the iso-elevation contours. Note that these contours, which are orthogonal in visual space, are no longer so when mapped onto the surface of the visual cortex. Note also that the magnification factor difference has disappeared and that the contour lines are more comparably spaced for these 2 axes. This finding holds true for all the animals that were examined. The preservation of cortical orthogonality for iso-elevation and isoazimuthal contours appears to be a special case, and suggests that this particular orthogonal coordinate system may have some special relevance for cortical processing in area 18 .

As a further illustration of this point, Figure $5 b$ shows the effect of representing the cortical map of visual space using a polar coordinate representation of receptive-field position. Now the continuous and dotted lines represent, respectively, constant (radial) distance (iso-eccentricity) and constant angle (anticlockwise-positive, starting at horizontal) relative to the area centralis. Note that this representation also fails to preserve orthogonality between real world contours and those of the cortical map. Since the horizontal magnification factor is so much greater than that for vertical (Fig. 4), the iso-eccentricity contours tend to run parallel to the iso-azimuthal contours of Figure 2. Likewise, the iso-angular contours (dotted lines in Fig. $5 b$ ) are "pulled" towards the iso-azimuthal contours of Figure 2 and thus the angle between the iso-eccentricity and iso-angular contours in Figure $5 b$ is substantially different from $90^{\circ}$.

\section{Disorder in the retinotopic map}

Scatter diagrams such as those in Figure 6 show that there is an average shift in receptive-field position with movement across the cortex, but they also suggest that, over a scale of a millimeter or so, the map deviates randomly from perfect smoothness. It is likcly that some of this deviation (i.e., the scatter relative to the regression line) is at least partly a consequence of the apparently random variation in receptive-field location observed when recording from successive cells during a penetration perpendicular to the cortical surface (Hubel and Wiesel, 1962, 1974a). We have expressed this scatter as twice the standard deviation of the distribution of receptive-field positions around a local mean of receptive-field position at each point in the map. We measured this mean by taking the average position of the receptive fields of points falling within a $0.5 \mathrm{~mm}$ radius of each particular point in the map. The distance from this mean of the receptive field of the point in question was then calculated, and the standard deviation of the resulting distribution measured. This was done separately for the elevational and azimuthal components of the scatter; it gave us a measure of receptive-field scatter, which was converted into cortical dimensions by multiplying by the magnification factors for elevation and azimuth, respectively. We refer to this scatter as the cortical point-spread function. It is a measure of the region of cortex within which a large proportion of neurons may be expected to have receptivefield centers in common. As this measure ignores receptive-field size, it must necessarily be smaller than the region within which cortical cells would have any part (not just the center) of their receptive fields in common.

The relationship between the cortical point scatter corresponding to the azimuthal and elevational components of receptive-field location for 11 different maps is shown in Figure $7 a$. It can be seen that the point scatter is always greater for the elevation component of receptive-field position than for the azimuthal component. This means that the point-spread func-
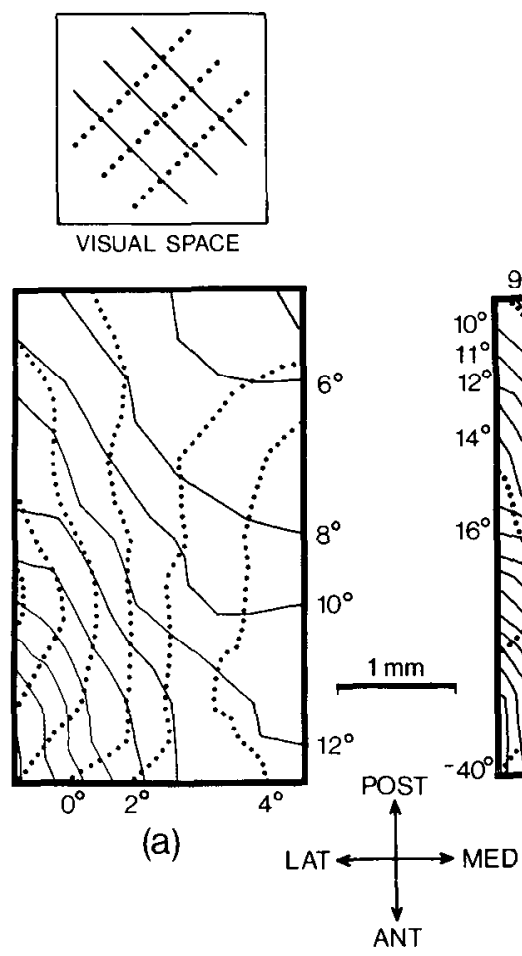

Figure 5. a, Illustration of how visual-field lines $45^{\circ}$ to the left and right of vertical are represented on the cortical surface. This is the same map shown on the left in Figure 2. $b$, Representation of polar coordinate visual-field lines on the surface of the visual cortex. Continuous lines are lines of constant radial distance (iso-eccentricity) in degrees of visual angle; dotted lines are lines of constant angle relative to horizontal.

tion is elongated along the anteropostcrior axis of thc cortex by a factor that varies over a range of 1.1 to 3.8 in different animals. The amount of elongation of the point-spread function was positively correlated with the cortical magnification factor anisotropy (Fig. $7 b$ ). Note, however, that the points in Figure $7 b$ fall systematically to the right of the dashed line, graphing equality between the asymmetries of the magnification factor and the point-spread function. The point-spread asymmetry is thus consistently less than that of the magnification factor anisotropy. Because of this, receptive-field scatter, expressed in terms of receptive-field position, is greater for azimuth than for elevation. Average scatters, expressed in degrees, were $4.1^{\circ}$ (range $2.6^{\circ}$ $6.0^{\circ}$ ) for azimuth and $2.75^{\circ}$ (range $1.6^{\circ}-4.7^{\circ}$ ) for elevation, with ratios (azimuth : elevation) varying over the range 1.06-2.17.

In Figure 8 we plot the relationships that the separate azimuthal and elevational components of receptive-field scatter have with cortical magnification factors for azimuth $(a)$ and elevation $(b)$, and with the mean azimuth $(c)$ and elevation $(d)$ of the individual maps. For the elevational component of the receptive field, no relationship at all is evident (Fig. 8, $b, d$ ), but the point-spread function is positively correlated with the magnification factor for azimuth (Fig. 8a). This means that the scatter of the map is greatest where the map is most fine-grained. Since the azimuthal magnification factor varies with horizontal eccentricity (Fig. 3), it is not surprising that there is a tendency, albeit a slight one, for the point-spread function for individual animals to vary inversely with mean horizontal eccentricity (Fig. $8 c$ ). Figure $8 d$ shows no strong relationship between the elevational component of the point-spread function and vertical eccentricity. 

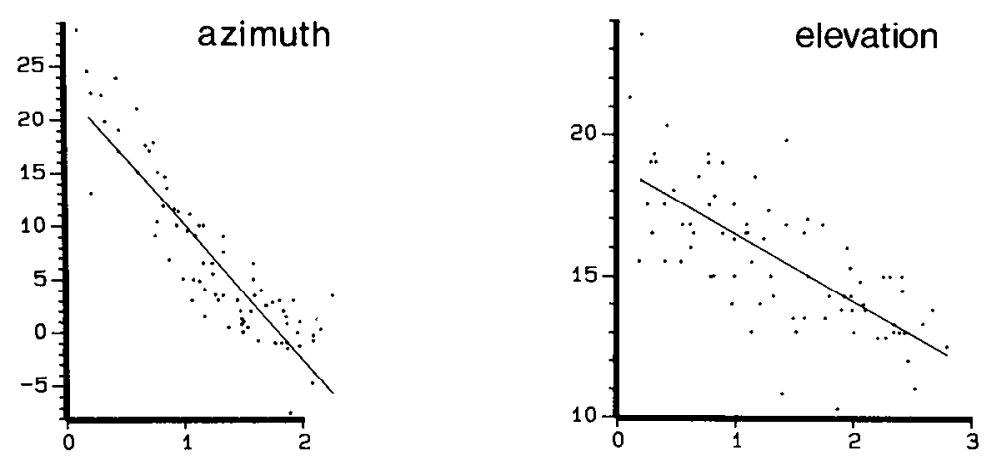

6SP
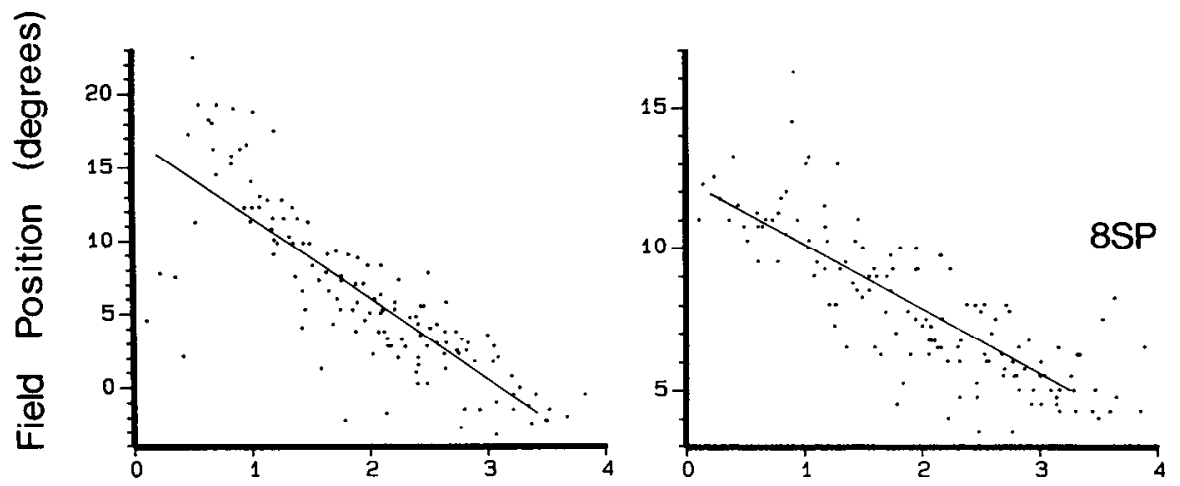

$\frac{1}{2}$
$\frac{2}{0}$
0
0
0
$\square$

Figure 6. Scatter plots of receptivefield-center position (elevation or azimuth) graphed against cortical distance for those angles of scan across the surface giving the best-fitting regression lines for 3 animals. Graphs on the left show how the azimuthal component of receptive-field position is correlated with distance across the cortex: graphs on the right show how the elevational component is correlated. Elevation units are in degrees below the horizontal meridian.
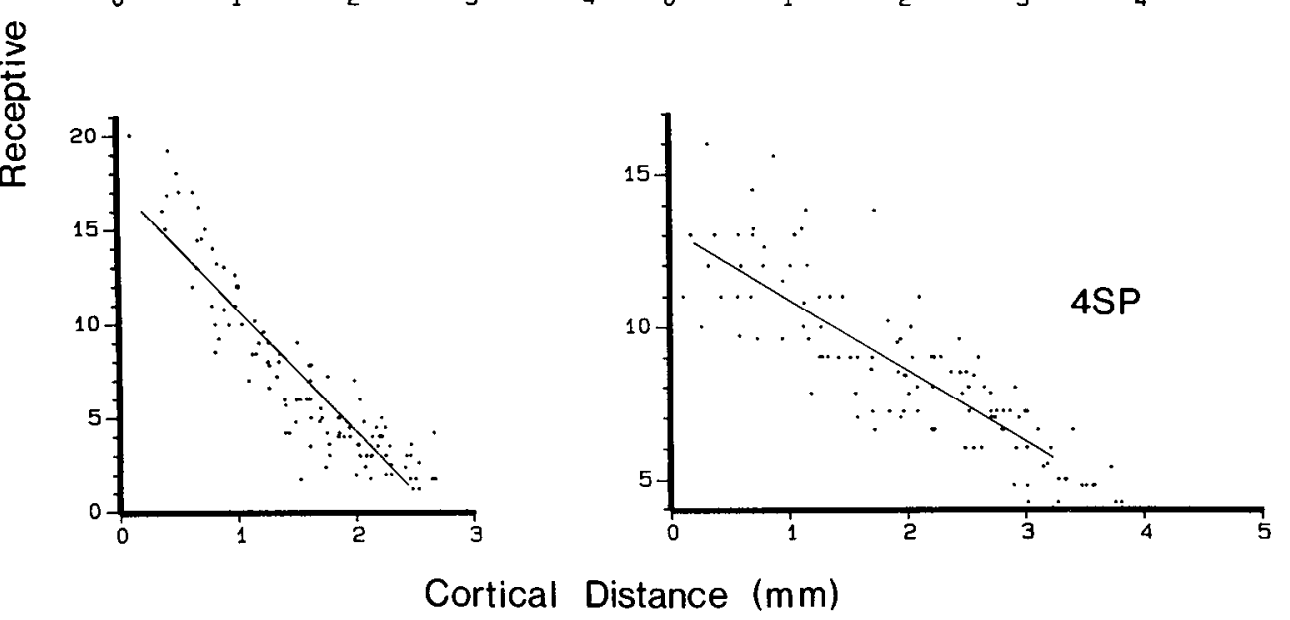

\section{Layout of response features}

In this section, we consider the tangential distribution in area 18 of units with similar orientation, direction, and ocular preferences. Figure 9 shows maps of preferred orientation for 2 different animals. The perspective of this figure (and that of Figs. 10,11,13) is looking down from above onto the cortex, and each point represents the result of 1 penetration. In Figure $13 a$ we show the layout of iso-orientation domains for a $90^{\circ}$ orientation range centered on vertical that was derived from the map on the right in Figure 9. To provide a more quantitative description of the periodicity and direction of elongation of the iso-orientation bands in this figure and in the other maps, the data were subjected to a 2-dimensional Fourier power spectrum analysis. A detailed description of the methods used and of the results obtained is given in an accompanying paper (Swindale et al., 1987). This analysis reveals 2 kinds of information: the overall periodicity of the iso-orientation bands, and the direction in which they tend to be elongated. In a 2-dimensional power spectrum, overall periodicity is determined by the distance of a peak in the energy profile from the origin, while the direction in which the iso-orientation bands run is orthogonal to the direction of the peak relative to the origin. This analysis revealed a remarkably constant periodicity in the different maps, averaging $1.25 \pm 0.13 \mathrm{~mm}$ in a total of 16 animals. The bands most commonly ran at an angle of about $25^{\circ}$ relative to the midline on the right-hand side of the brain, as viewed in Figure 1 , i.e., sloping from posteromedial to anterolateral. Determination of this angle, however, was sometimes complicated by the presence of 2 peaks or of energy spread over a range of directions relative to the origin (although always at the same distance from it). Quitc probably this variability was due to the relatively small size of the maps, which often included only 2 or 3 complete cycles of orientation.

Figure 10 illustrates the arrangements of units with similar ocular preferences in 2 representative animals. Here the letters $\mathrm{C}$, I, or B represent clusters of cells driven strongly via the contralateral or ipsilateral eyes, or via both eyes. The maps 


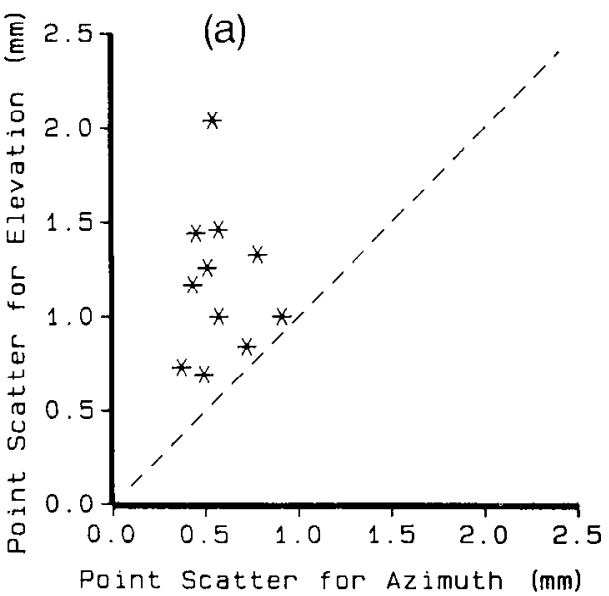

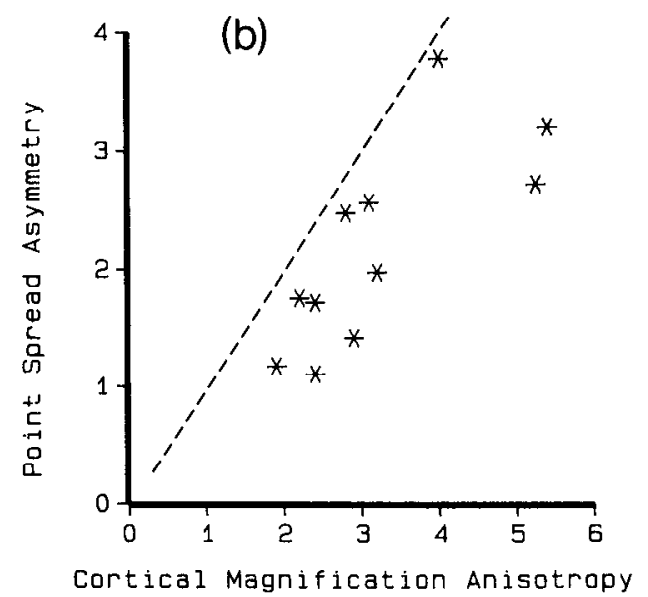

Figure 7. a, Scatter graph showing the relation of the azimuthal and elevational components of receptive-field scatter in each of 11 different animals. $b$, Point-spread asymmetry, i.e., the ratio between the 2 components of receptive-field scatter (elevation: azimuth) graphed against the ratio between the magnification factors for elevation and azimuth. Dashed lines graph equality between the measured parameters. confirm many other reports (e.g., Hubel and Wiesel, 1962, 1963) showing that neurons with similar ocular preference are arraycd in clusters across the cortical surface. The clusters, however, show little tendency to form bands. We represented ocular dominance at each recording site on a scale from 1 to 7 (Hubel and Wiesel, 1962) and subjected the data to periodicity analysis, similar to that described above for the orientation data. The ocular dominance power spectra confirmed our visual impression that the ocular dominance aggregates were not strongly elongated. The peaks in the spectra were of near equal size regardless of their direction relative to the origin. The periodicity of the ocularity aggregates averaged $1.86 \pm 0.75 \mathrm{~mm}$. This spacing was significantly grcater than that for oricntation $(p<$ 0.05 ; Mann-Whitney test), as well as being more variable from animal to animal (Fig. 12).

The evidence of columnar organization for the preferred direction of motion comes from studies where long penetrations have been made perpendicular to the cortical surface in area 18. These show that it is common for neighboring units to have the same preferred direction, as well as the same preferred orientation and eye input (Payne et al., 1981; Tolhurst et al., 1981). When we recorded unit clusters in our mapping experiments,
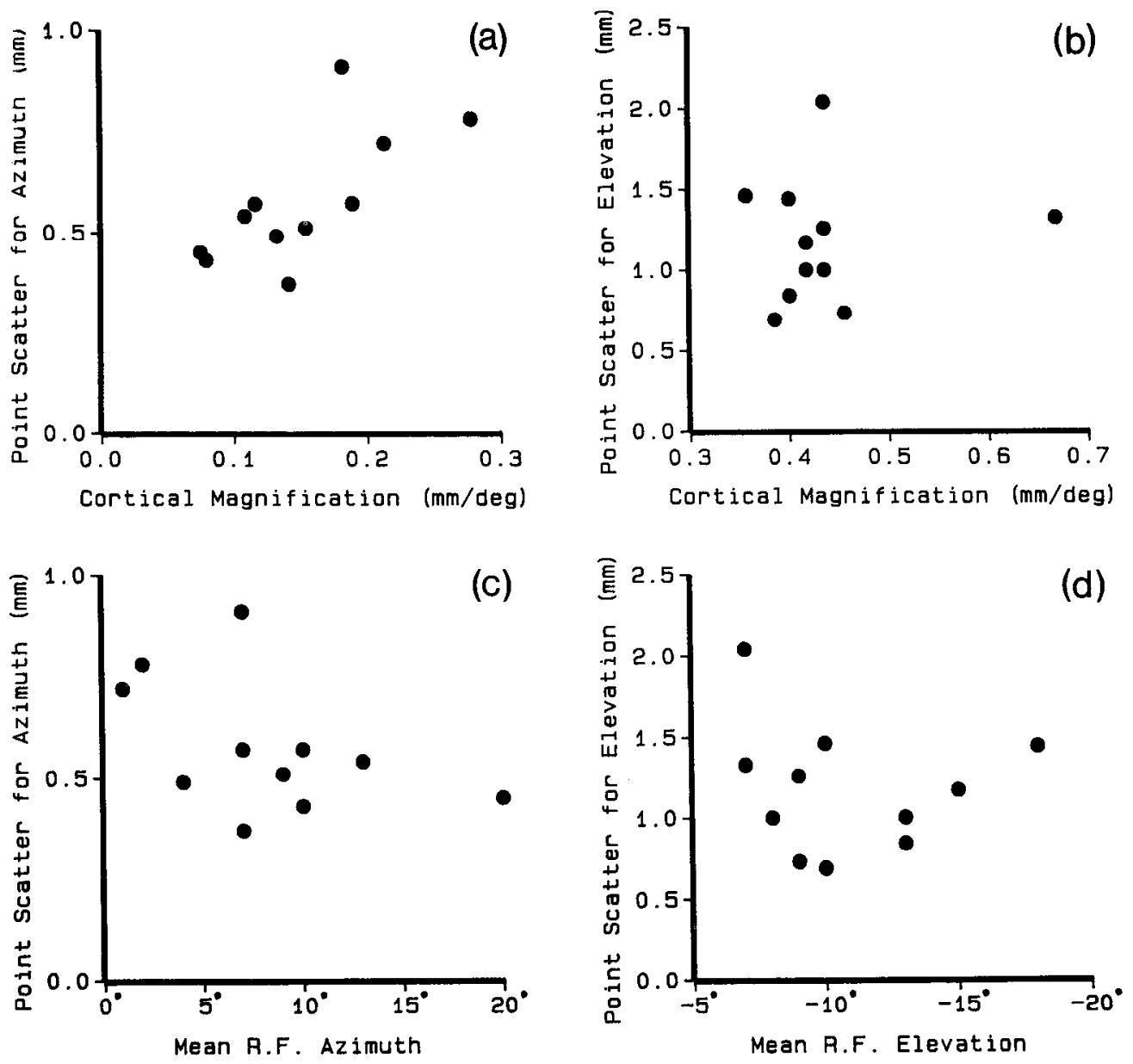

Figure 8. Graphs of the 2 components of receptive-field scatter: azimuthal and elevational against cortical magnification factor for azimuth $(a)$ and elevation $(b)$, and against the mean azimuthal $(c)$ and elevational $(d)$ positions of maps in 11 different animals. 
Figure 9. Maps of preferred orientation from 2 different animals. Each point represents 1 recording locus. Asterisk marks the location of a nonoriented response.
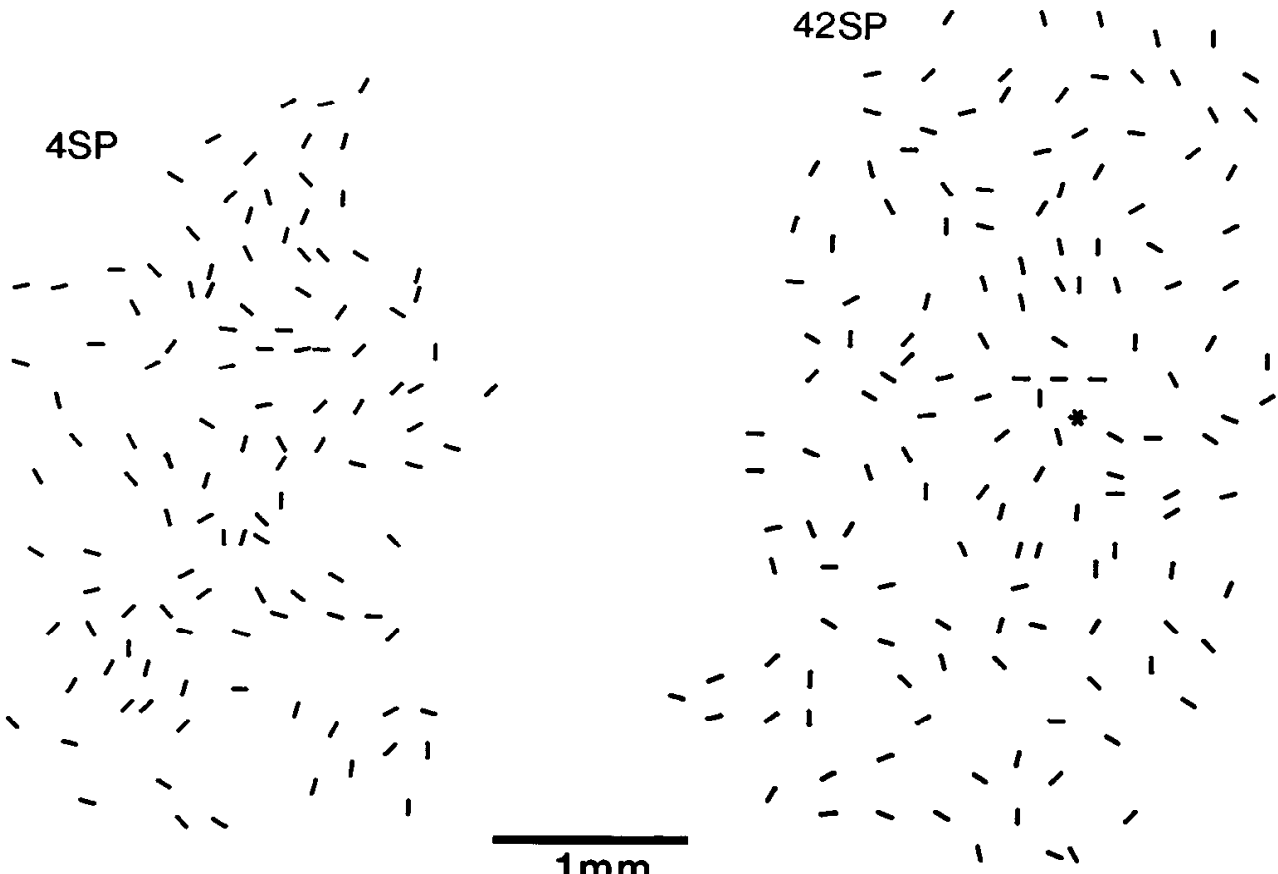

we frequently found that the entire cluster showed the same preferred direction. Maps of direction preference are shown for 2 representative maps in Figure 11. There is a tendency, evident in Figure 11, and shown more clearly by autocorrelation analysis, for units $300 \mu \mathrm{m}$ or less apart to exhibit a similarity in their directional preferences (Swindale et al., 1987). In some of the maps, it was possible, on the basis of visual inspection and with the help of interpolated maps of orientation preference, to define local clusters of points with similar direction preference, with boundaries defined by either a continuous change in orientation or a discontinuous change in direction preference (Fig. $13 c$; fig. 15 in Swindale et al., 1987). No clear peak in the power

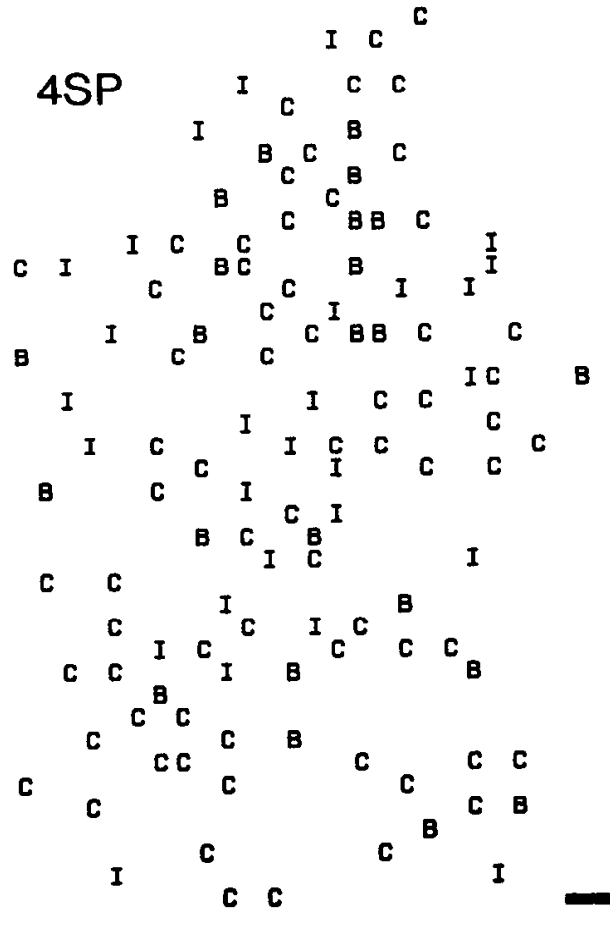

$1 \mathrm{~mm}$

Figure 10. Maps of ocular dominance from 2 animals. $C$, Points in ocular dominance categories $1-3 ; B$, points in category 4 ; $I$, points in categories 5-7. 


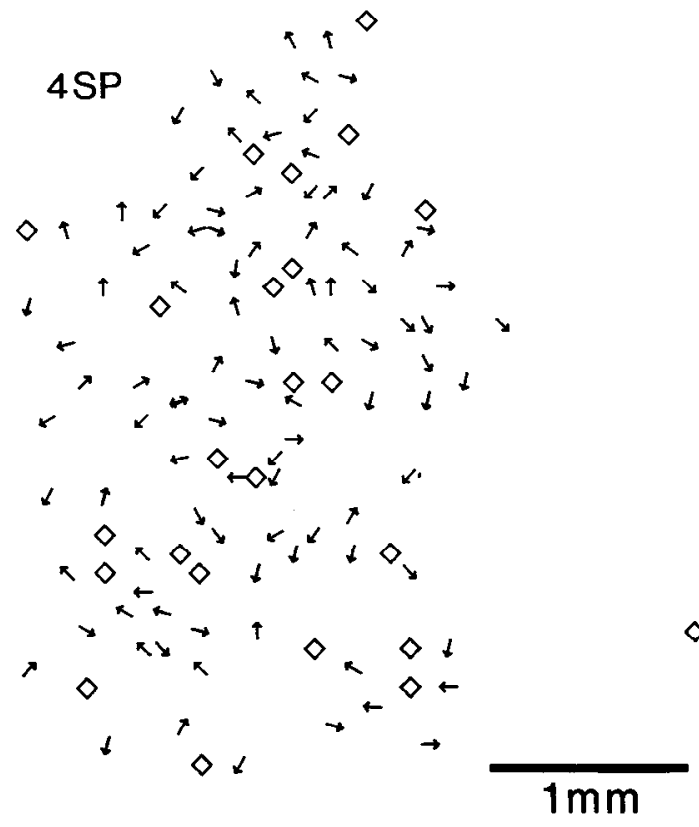

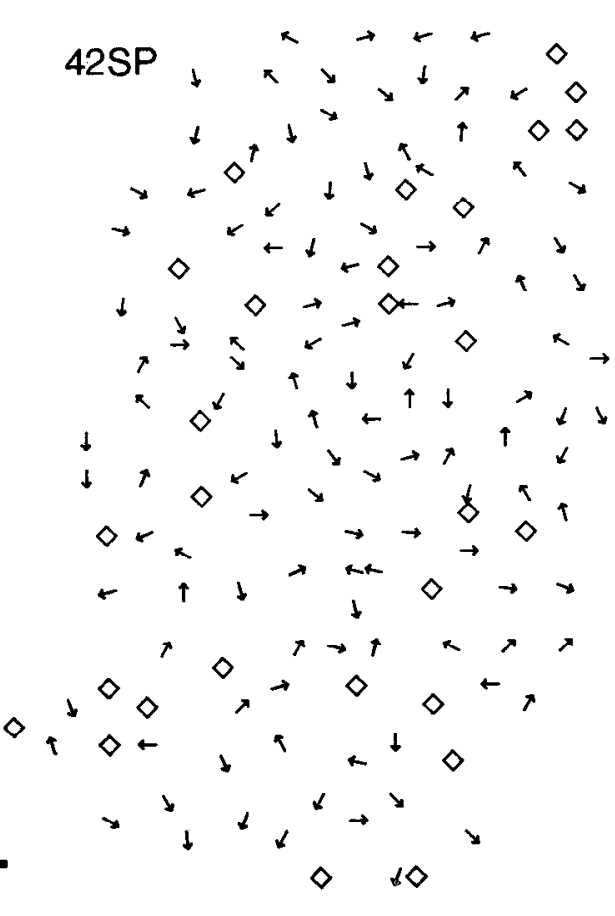

Figure 11. Maps of preferred direction from 2 different animals. Diamonds are points that lacked clear direction selectivity. spectrum of direction preference was ever apparent (Fig. 11), although this does not rule out the existence of a systematic periodic organization of direction preference on a larger scale. One cause for the absence of any form of organization, as shown by Fourier analysis, may be the presence of many $180^{\circ}$ reversals in direction preference. These would tend to disrupt continuity in the direction map, on which the presence of a peak in the power spectrum depends.

\section{Relationships among response properties and topographic maps}

The foregoing results have shown that an arrangement of feature-specific responses is superimposed on an inexact anisotropic map of the visual field in area 18. How are these functional maps related to each other? To approach this issue we show summary maps for orientation, ocularity, and direction preferences for 1 representative animal in Figure 13 (upper panels). In the lower panels of Figure 13 we show the results of superimposing the different maps. These various superpositions show how the intersections of different sets of feature-specific neurons are represented across the cortical surface. The ellipse in the lower right-hand corner of Figure 13 shows the approximate shape of the cortical point-spread function; it is evident that the representation of most stimulus combinations would be very much better in some visual-field locations than in others. Even for fairly broadly defined stimulus combinations (e.g., that in Fig. 13f, with stimuli moving upwards and to the left, as seen through the ipsilateral eye) there would be visual-field locations in which such stimuli would scarcely be seen by the cortex at all.

No obvious structural relationships were observed between the orientation and direction bands and the ocular dominance patches. The iso-orientation domains tended to run across the cortex in a direction approximately orthogonal to the axis of elongation of the point spread-function and in the direction of the iso-azimuthal contours.

\section{Discussion}

\section{Retinotopic map and point-spread function}

The retinotopic organization of area 18 has been studied, using physiological mapping techniques, by Albus and Beckman (1980) and Tusa et al. (1979). Our maps cover a much more restricted cortical area than do theirs, but the grain of our maps is considerably finer. Over the area of cortex that we examined, the retinotopic maps are continuous and have markedly unequal magnification for horizontal and vertical space. This distortion, the exact size of which varies from map to map in individual animals, has the effect of increasing the amount of cortical territory devoted to a given vertical distance in visual space relative to horizontal distance.

The unequal magnification for horizontal and vertical space is evident in the earlier coarse-grain maps, but these show great individual variability, "islands" of vertical meridian representation (Sanides and Albus, 1980), and other features that are not a prominent part of our data. Our maps deliberately cover a restricted and easily accessible part of the cortex, and may not be large enough to reveal some of these features. The advantages of making a confined map based on many closely spaced penetrations in a region of cortex that is flat are several. The high density of data points makes it possible to study in detail the local relationship between the mapping of iso-elevation and isoazimuthal contours across the cortical surface, and to measure the amount by which the map deviates locally from smoothness and continuity. Because the region of cortex that we studied was approximately flat, errors associated with reconstruction of penetrations in sulci were avoided, and the special methods necessary for representing and studying maps on curved cortical surfaces (Van Essen and Maunsell, 1980) were not needed.

It is striking that the magnification factor difference is greatest for the representation of horizontal and vertical axes in visual space, with no obvious inequality present when the map contour lines represent visual-field lines $45^{\circ}$ to the right and left of ver- 

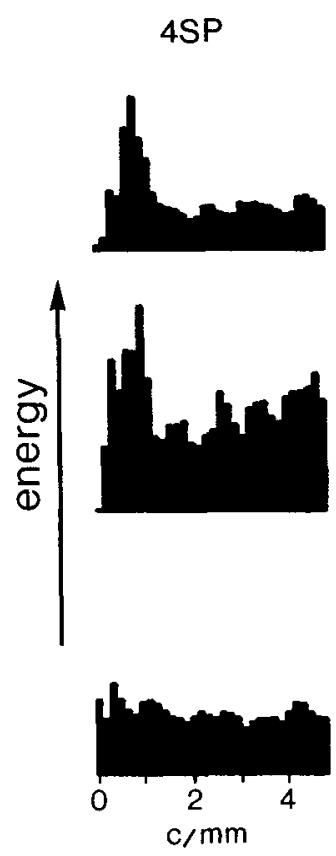
a $180^{\circ}$ range of directions for a given
distance relative to the origin, normalizing energy to unit area in frequency space.

Figure 12. Fourier power spectra (energy vs spatial frequency) for 3 maps of orientation preference (upper row), ocular dominance (middle row), and direction selectivity (bottom row). The horizontal scale is in cycles per millimeter. The origin (i.e., zero frequency or DC) is the leftmost bin in each histogram. The spectra were obtained from 2-dimensional transforms by averaging over mensional transforms by averaging over

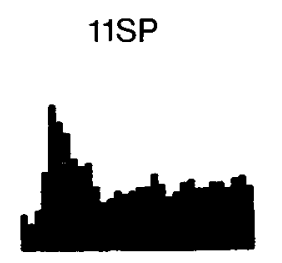

tical (Fig. 5). The functional significance of this unequal magnification for horizontal and vertical space remains uncertain. One possibility is that the unequal magnification factor relates to binocular vision. Because of the horizontal offset of the 2 eyes, the images of objects that are not on the fixation plane would be located at noncorresponding, horizontally separated loci on the 2 retinas. Vertical image misalignments between the eyes also occur when an object is closer to one eye than the other, but these misalignments are not related to convergence position and are much smaller than the range of horizontal misalignments seen (Ogle, 1950; Mayhew and Longuet-Higgins, 1982). Since both horizontal and vertical image misalignments are useful for binocular vision (Mayhew and Longuet-Higgins, 1982), the unequal magnifications and the scatter of horizontal and vertical space representation in the cortex may afford the opportunity for the detection of relatively large horizontal misalignments between the 2 eyes' inputs to a given cortical cell, as well as that of relatively small vertical misalignments.

A second, possibly related, function of the topographic anisotropy in area 18 may relate to the foreshortening of the visual world along the vertical dimension that occurs when a cat gazes at level terrain. Since the animal views the ground from an oblique angle, the vertical dimension of the terrain will be compressed relative to the horizontal dimension. From this perspective, the unequal magnification of horizontal and vertical retinal angles is seen as a mechanism that compensates for the retinal foreshortening, and arranges a more isotropic representation of the actual terrain, rather than its foreshortened image, on the cortical surface.

It might be argued that the anisotropic magnification of visual space in area 18 has no function at all, and that it results simply from the fact that area 17 and 18 border each other along the vertical meridian representation. This abutment constrains the magnification factor for vertical space to be equal in the 2 areas, at least for the area near the vertical meridian representation. Since area 18 is narrow in shape, the unequal magnification could simply result from the elongated shape of the structure as a whole. If this is true, then both the elongated point-spread function and the greater spread of intrinsic connections (Matsubara et al., 1987) along the anterior-posterior axis of the gyrus may represent attempts by the cortex to correct for the anatomical asymmetry and to restore a functional symmetry in cortical properties related to visual space. It is equally plausible, of course, that the elongated shape of area 18 is a consequence of some more important constraint that is a primary cause of the unequal magnification of the retinotopic map in this structure.

The mechanisms underlying the unequal magnification factors must be sought in the geniculostriate mapping. Certainly the retinal map itself is isotropic. At the level of the lateral geniculate nucleus (LGN), one observes a small anisotropy in the visual-field map that accentuates the representation of vertical space (fig. 8 in Sanderson, 1971). This anisotropy varies from place to place in the LGN, however, and is always much less than that reported here for area 18 . Thus, to account for the anisotropy in the cortical map, incoming geniculate axons would have to have a greater average spacing in the A-P than in the M-L direction. There is as yet no direct evidence for this. A tendency for individual LGN terminal arbors in area 18 to extend further along the A-P cortical axis than along the M-L axis has been noted, however (IIumphrey et al., 1985). This asymmetric termination pattern would not in itself cause an unequal magnification of the cortical map, but might well contribute to the existence of an elongated point-spread function.

The point-spread function, as we have defined it, is a statistical measure of the deviation of the retinotopic map in the cortex from perfect smoothness. Receptive-field locations appear to be approximately normally distributed around the local mean of receptive-field position. We express the magnitude of the scatter as being twice the standard deviation of this distribution. This scatter can be measured either in terms of receptive-field position or, if transformed via magnification factor, in terms of cortical distance.

In the first case, the measurement expresses the distribution in space of receptive-field centers associated with a single cor- 

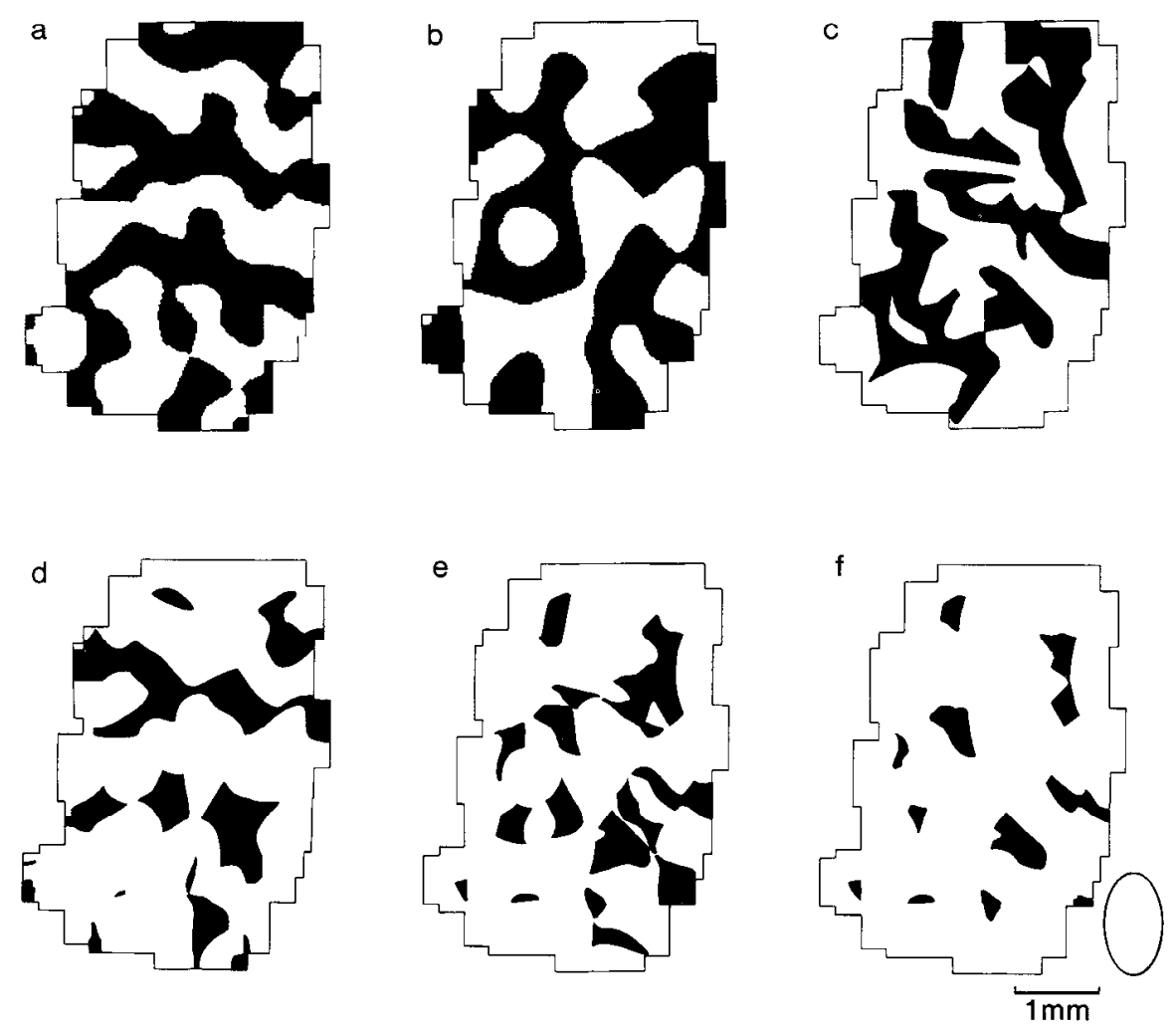

Figure 13. $a-c$, Summary maps of the layout of orientation, ocular dominance, and direction preference for 1 representative animal. a, Shaded areas show the distribution of orientations lying in a $90^{\circ}$ range centered on vertical. $b$, Shaded areas show regions of cortex receiving strong ipsilateral eye input (O.D., groups 4-7).c, Shaded areas show the distribution of direction selectivities that are upwards and to the left, i.e., in a $180^{\circ}$ range from $45^{\circ}$ to $225^{\circ}$. $d-f$. Superpositions of the 3 maps $(a-c)$ were made to show how some combinations of response features were represented on the cortex. $d$, Vertical orientations (range, $45^{\circ}-135^{\circ}$ ) driven by the ipsilateral eye. $e$, Regions with upward direction selectivity $\left(0^{\circ}-180^{\circ}\right)$ driven by the ipsilateral eye. $f$, Regions with direction selectivities in a $90^{\circ}$ range upwards and to the left, driven by the ipsilateral eye. The ellipse shows the approximate size of the cortical pointspread function measured for area 18 . tical location. This scatter averaged $4.1^{\circ}$ horizontally by $2.75^{\circ}$ vertically, and was roughly equal to receptive-field size, which normally lay in the range $2^{\circ}-6^{\circ}$. It should be remembered that because these measurements are standard deviations, about a third of the relevant observations can be expected to fall outside these limits. Equivalence between receptive-field size and receptive-field scatter was first proposed by Hubel and Wiesel (1974b) for area 17 of the monkey, and later by Albus (1975a) for area 17 of the cat.

When expressed in terms of cortical distance, the point-spread function gives a measure of the distribution in the cortex of cells whose receptive-field centers would be stimulated by a particular small region in visual space. This distribution was always elongated along the A-P axis, and varied within the range 0.4-0.9 $\mathrm{mm}$ along the $\mathrm{M}-\mathrm{L}$ axis and $0.7-2.0 \mathrm{~mm}$ along the A-P axis (Fig. 7a). The degree of anisotropy in the point-spread function was positively correlated with the degree of anisotropy in the retinotopic map (Fig. $7 b$ ), although the map was always more anisotropic than the point-spread function. The spread along the M-L axis was also correlated with cortical magnification (Fig. 8a), being greatest where the magnification factor was greatest, i.e., çlose to the vertical meridian representation, medial on the gyrus. No correlation between the spread along the A-P axis and the cortical magnification factor for elevation was observed (Fig. $8 b$ ), perhaps because the overall range of cortical magnifications involved was small. These observations show that the cortical point-spread function can vary in size within a single cortical area, and that it gets smaller with increasing receptive-field eccentricity. A similar correlation has been discovered in area 17 of the monkey by Dow et al. (1981) and Van Essen et al. (1984).

At first glance, it might appear to be desirable to keep the point-spread function as small as possible. This would result in a direct and precise relationship between cortical and retinal locations and would be expected to greatly facilitate such tasks as visual localization and 2-point discrimination. It is possible to construct broadly tuned representations of space (i.e., with large point-spread functions) that are capable of accurately localizing a single object (Hinton, 1984), but such distributed representations fail when the location of several different closely spaced objects must be coded at the same time. Why, then, is the point-spread function as large as it is, and why is it anisotropic?

It is likely that the size and shape of the point-spread function can best be understood in terms of the mechanism by which cortical maps manage to represent a variety of different features of the visual stimulus at each point in the visual field (Hubel and Wiesel, 1974a). Figure 13 (upper panels) shows summary maps for orientation, ocular dominance, and direction preference for 1 representative animal. The lower panels of Figure 13 show the results of superimposing the different maps. These various superpositions illustrate how the intersection of different feature sets is represented across the cortical surface, and highlight the difficulty involved in representing all combinations of orientation, direction, and eye preference within an area of cortex representing a given point in visual space. Thus there are a number of regions in the lower panels of Figure 13 where certain combinations of features are either apparently not represented at all, or less often than in other parts of the map. This lack suggests a diminished capacity on the part of area 18 to accurately represent certain properties of the visual world for certain regions in visual space. The problem would be aggravated if, as seems probable, stimulus features other than the 3 considered here had to be represented, and especially if these properties had a representation on the cortex that was also orderly, periodic, and columnar. 
The cortex may adopt a number of different strategies to solve this problem in representation. When 2 columnar systems are highly periodic and stripe-like, one solution would be for the stripes to intersect at right angles (Hubel and Wiesel, 1977). There is some evidence for local orthogonality between isospatial frequency domains and iso-orientation domains in the monkey (Tootell et al., 1982), although there is no evidence for this kind of organization in the cat visual cortex. Another strategy that would tend to maximize overlap would be for the periodicities of independent columnar systems to be different, so as to avoid destructive "beating" of the representations. Differences in the periodicities of orientation and ocular dominance bands appear to be the rule in both cat and monkey visual cortex. In our data from cat area 18 , the periodicities of ocular dominance and orientation domains were 1.86 and $1.25 \mathrm{~mm}$, respectively - a ratio of about 1.5 . In monkey area 17 , the periodicities have a similar ratio (1.4), being 0.8 and $0.57 \mathrm{~mm}$ for ocular dominance and orientation, respectively (Hubel and Wiesel, 1974b; LeVay et al., 1975). Another strategy would be for some representations to be less orderly than others, or even random. The representation problem would largely disappear if all properties were randomly and independently distributed in the cortex, since there are probably sufficient numbers of neurons for all combinations to be represented on the basis of chance. It seems possible, although it is not yet known, that some rcsponse properties, such as preferred velocity or length selectivity, might have a random distribution across the cortical surface. The fact that at least some of the representations are orderly indicates that there is some special advantage in maximizing continuity. Finally, increasing the size of the pointspread function would, by increasing the allocation of cortical volume to the analysis of each retinal location, increase the completeness of the representation, though presumably at the expense of accuracy in cortical localization and 2-point resolution. The increase in the size of the point-scatter function with increasing closeness to the vertical meridian representation, observed by us in area 18 of the cat and by Dow et al. (1981) and Van Essen et al. (1984) in area 17 of the monkey, might therefore be one mechanism by which the cortex would be able to analyze central visual-field locations more completely than peripheral ones. The presumed decrease in the accuracy of cortical localization would be more than compensated for in retinal tcrms by the increase in magnification factor for central visual fields.

Our maps are all made at about the same depth in the cortex $(400-700 \mu \mathrm{m})$, and thus our measurements are accurate only for that depth. However, there is evidence that, in monkey visual cortex, receptive-field scatter is not equal in all layers, topographic precision being greater in layer IV than in the upper and lower layers (Hubel and Wiesel, 1974a; Hubel et al., 1974). The same may well be true in the cat. This would imply a smaller point-spread function in this layer and suggests that the set of response features that must be represented may be smaller in layer IV than in other layers. This is consistent with the lack of orientation selectivity found in cells in layer IV in the monkey (Hubel and Wiesel, 1974b).

The point-spread function that we observe may thus represent a compromise between the contradictory demands for high precision of localization of multiple stimuli and representation of many response features for each point in visual space (Hubel and Wiesel, 1974a). Consequently, the elongation of the pointspread function observed along the A-P axis may have a number of functions. As suggested above, it may represent an attempt by the cortex to compensate for the anisotropic retinotopic map, so that the point-spread function expressed in visual-field coordinates is more circular than it would be otherwise. Another possibility is that the direction of elongation is related to the direction in which the iso-orientation domains run. These 2 directions are roughly orthogonal, and this would tend to maximize the variation of orientation preference within the pointspread function, thus maximizing coverage of this parameter.

\section{References}

Albus, K. (1975a) A quantitative study of the projection area of the central and paracentral visual field in area 17 of the cat. I. The precision of the topography. Exp. Brain Res. 24: 159-179.

Albus, K. (1975b) A quantitative study of the projection area of the central and paracentral visual field in area 17 of the cat. II. The spatial organisation of the orientation domain. Exp. Brain Res. 24: 181-202.

Albus, K. (1979) ${ }^{14} \mathrm{C}$-deoxyglucose mapping of orientation subunits in the cat's visual cortical areas. Exp. Brain Res. 37: 609-613.

Albus, K., and R. Beckman (1980) Second and third visual areas of the cat: Interindividual variability in retinotopic arrangement and cortical location. J. Physiol. (Lond.) 299: 247-276.

Bishop, P. O., G. H. Henry, and C. J. Smith (1971) Binocular interaction fields of single units in the cat striate cortex. J. Physiol. (Lond.) 216: 39-68.

Chow, K. L. (1968) Influences of residual eye movements in single unit studies of the visual system. Brain Res. 8: 385-388.

Cynader, M., and D. E. Mitchell (1980) Prolonged sensitivity to monocular deprivation in dark-reared cats. J. Neurophysiol. 43: 10261040 .

Cynader, M., J. C. Gardner, and M. Mustari (1984) Effects of neonatally induced strabismus on binocular responses in cat area 18. Exp. Brain Res. 53: 384-399.

Dow, B. M., A. Z. Snyder, R. G. Vautin, and R. Bauer (1981) Magnification factor and receptive field size in foveal striate cortex of the monkey. Exp. Brain Res. 44: 213-228.

Hinton, G. E. (1984) Distributed representations. Technical report CMI J-CS-84-157, Dept. of Computer Science, Pittsburgh, PA.

Hubel, D. H., and T. N. Wiesel (1962) Receptive fields, binocular interaction and functional architecture in the cat's visual cortex. J. Physiol. (Lond.) 160: 106-154.

Hubel, D. H., and T. N. Wiesel (1963) Shape and arrangement of columns in cat's striate cortex. J. Physiol. (Lond.) 165: 559-568.

Hubel, D. H., and T. N. Wiesel (1974a) Uniformity of monkey striate cortex: A parallel relationship between field size, scatter and magnification factor. J. Comp. Neurol. 158: 295-306.

Hubel, D. H., and T. N. Wiesel (1974b) Sequence regularity and geometry of orientation columns in the monkey striate cortex. J. Comp. Neurol. 158: 267-294.

Hubel, D. H., and T. N. Wiesel (1977) Functional architecture of macaque monkey visual cortex. Proc. R. Soc. Lond. [Biol.] 198: 159.

Hubel, D. H., T. N. Wiesel, and S. LeVay (1974) Visual field representation in layer IVc of monkey striate cortex. In Society for Neuroscience Program and Abstracts (4th Annual Meeting), p. 264.

Hubel, D. H., T. N. Wiesel, and S. LeVay (1977) Plasticity of ocular dominance columns in monkey striate cortex. Phil. Trans. R. Soc. Lond. [Biol.] 278: 131-163.

Hubel, D. H., T. N. Wiesel, and M. P. Stryker (1978) Anatomical demonstration of orientation columns in macaque monkey. J. Comp. Neurol. 177: 361-380.

Humphrey, A. L., L. C. Skeen, and T. T. Norton (1980) Topographic organisation of the orientation column system in the striate cortex of the tree shrew (Tupaia glis). II. Deoxyglucose mapping. J. Comp. Neurol. 192: 549-566.

Humphrey, A. L., M. Sur, D. J. Uhlrich, and S. M. Sherman (1985) Termination patterns of individual $X$ - and $Y$-cell axons in the visual cortex of the cat: Projections to area 18, to the $17 / 18$ border region, and to both areas 17 and 18. J. Comp. Neurol. 233: 190-212.

Kennedy, C., M. H. Des Rosiers, O. Sakurada, M. Shinohara, M. Reivich, H. W. Jehle, and L. Sokoloff (1976) Metabolic mapping of the primary visual system of the monkey by means of the autoradio-

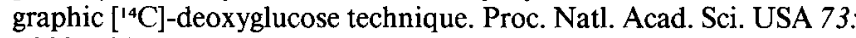
$4230-4234$. 
Levay, S., D. H. Hubel, and T. N. Wiesel (1975) The pattern of ocular dominance columns in the macacque visual cortex revealed by a reduced silver stain. J. Comp. Neurol. 159: 559-576.

Matsubara, J. A., M. S. Cynader, and N. V. Swindale (1987) Anatomical properties and physiological correlates of the intrinsic connections in cat area 18. J. Neurosci. 7: 1428-1446.

Mayhew, J. E. W., and H. C. Longuet-Higgins (1982) A computational model of binocular depth perception. Nature 297: 376-378.

Ogle, K. N. (1950) Researches in Binocular Vision, Saunders, Philadelphia, PA.

Payne, B. R., N. Berman, and E. H. Murphy (1981) Organisation of direction preferences in cat visual cortex. Brain Res. 211: 445-450.

Sanderson, K. J. (1971) Visual field projection columns and magnification factors in the lateral geniculate nucleus of the cat. Exp. Brain Res. 13: 159-177.

Sanides, D., and K. Albus (1980) The distribution of interhemispheric projections in area 18 of the cat: Coincidence with discontinuities of the representation of the visual field in the second visual area (V2). Exp. Brain Res. 38: 237-240.

Schwartz, E. L. (1980) Computational anatomy and functional architecture of striate cortex: A spatial mapping approach to perceptual coding. Vision Res. 20: 645-669.

Shatz, C. J., S. Linström, and T. N. Wiesel (1977) The distribution of afferents representing the right and left eyes in the cat's visual cortex. Brain Res. 131: 103-116.

Singer, W. (1981) Topographic organisation of orientation columns in the cat visual cortex. Exp. Brain Res. 44: 431-436.
Swindale, N. V., I. A. Matsubara, and M. S. Cynader (1987) Surface organization of orientation and direction selectivity in cat area 18 . J. Neurosci. 7: 1414-1427.

Tolhurst, D. J., A. F. Dean, and I. D. Thompson (1981) Preferred direction of movement as an element in the organisation of cat visual cortex. Exp. Brain Res. 44: 340-342.

Tootell, R. B. H., M. S. Silverman, E. Switkes, and R. L. DeValois (1982) The organisation of cortical modules in primate striate cortex. Soc. Neurosci. Abstr. 8: 707.

Tusa, R. J., A. C. Rosenquist, and L. A. Palmer (1979) Retinotopic organisation of areas 18 and 19 in the cat. J. Comp. Neurol. 185: $657-678$.

Van Essen, D. C., and J. H. R. Maunsell (1980) Two-dimensional maps of the cerebral cortex. J. Comp. Neurol. 191: 255-281.

Van Essen, D. C., W. T. Newsome, and J. H. R. Maunsell (1984) The visual field representation in striate cortex of the macaque monkey: Asymmetries, anisotropies and individual variability. Vision Res. 24 $429-448$.

Wiesel, T. N., D. H. Hubel, and D. M. K. Lam (1974) Autoradiographic demonstration of ocular dominance columns in the monkey striate cortex by means of transneuronal transport. Brain Res. 79 . 273-279.

Wolbarsht, M. C., E. F. MacNicol, and H. G. Wagner (1960) Glass insulated platinum microelectrode. Science 132: 1309-1310. 